\title{
Definition of Key Indicators to Identify Optimal Distribution Grid Restoration Strategies
}

\author{
Christoph Strunck and Christian Rehtanz \\ TU Dortmund, Institute for Energy Systems, Energy Efficiency and Energy Economics, 44227 Dortmund, Germany
}

\begin{abstract}
Due to significant changes in the power energy system and extreme weather conditions as a result of the increasing impact of climate change, large scale blackouts become more likely. With the rising penetration of renewable energy sources in distribution grids and the shutdown of large conventional power plants, the system inertia and therefore the resilience is decreasing. This will have a significant influence on the provision of ancillary services in the future. Especially for grid restoration processes, new concepts are necessary to assure an optimal integration of the distributed energy resources to resupply a grid after a blackout. However, to identify and assess the capability of distribution grids to restore the grid operation to resupply their grid independently of the transmission system key indicators are necessary for an analysis. Hence, this paper introduces a key indicator system, which has the goal to address several challenges of a distribution grid restoration.
\end{abstract}

\section{Motivation}

To reach the global goals of carbon reduction, to minimize the negative effects of climate change, especially the energy systems has begun to change in the last years. In the electrical power system, these changes are mainly on the generation side. Huge nuclear and coal power plants are going to be shut down and replaced by smaller decentralized energy resources (DER) units like wind power turbines and solar power plants. This poses several challenges for the grid operation in the future. On the one hand, these power plants are fluctuating generation units and therefore less reliable. Moreover, they are not necessarily near load centres, but rather distributed and connected to the geographically optimal place in the distribution system. On the other hand, DERs are mostly connected via converter. Hence, the inherent inertial reaction of the synchronous generators that provide additional stability and resilience to the grid is no longer available. [1] In the future, more system splits and blackouts can be expected, as it can already be observed today due to e.g. extreme weather situations $[2,3]$. However, DERs offer new opportunities for new innovative strategies like a grid restoration of distribution grids and the stable and reliable island grid operation of such grids.

Already today, many distribution grids, especially in Europe, theoretically have the potential to perform a grid restoration with DERs. [4] However, sophisticated strategies and investigations are necessary to identify the best grid restoration strategy as well as to assess the critical steps during the process. Obviously, additional communication and digitalization as well as the enhancement of control schemes of larger DERs will improve the capability to be able to blackstart the grid. A considerable amount of research is focusing on the possibility of the restoration process based on quasi- dynamic analyzations that are considering the net balance of demand and generation with DER and battery energy storage systems (BESS) under different weather and load conditions [4-6]. However, in future research it will be important to evaluate grid restoration processes in a distribution grid with dynamic studies, which poses several challenges. [7]

Hence, to validate not only the dynamic ability for a distribution grid operator to restore its grid, but also the best restoration strategy, key indicators are necessary. In this paper, different indicators are presented and discussed with regard to the restoration process and the subsequent island grid operation. The factors are classified and further analysed in respect to possible strategies or recommendations for such. To build up the restoration process indicators, available data of distribution grid operators will be considered. This includes but is not limited to the annual peak load $P_{\text {load,max }}$, the number of grid areas $n_{\text {gridarea }}$, the number of remote controllable circuit breakers, and the installed PV $P_{P V}$ or DER $P_{D E R}$ power, respectively. This data should be available as part of the published grid structure data which are published by every European distribution system operator (DSO) yearly [8]. However, more detailed information like the share of remote-controlled circuit breakers and grid areas are not publicly available. The better the database, the better the grid operator can be given an indication of the blackstart capability of the grid and which additional measures the operator may have to consider.

This paper is structured as follows. In section 2, the challenges of a blackstart of a distribution grid is addressed. Based on these challenges, the developed key indicators are presented in chapter 3. In chapter 4, the indicator system will be applied on a real German distribution system before chapter 5 concludes the paper and gives an outlook. 


\section{Challenges of a distribution grid restoration process}

In case of a grid restoration process in a distribution grid with a high share of renewable energy sources (RES), there are different challenges compared to a conventional grid restoration process. For once, blackstart units (BSUs) with suitable control schemes are needed.

Moreover, a significant part of the available RES power comes from distributed PV plants, which cannot be controlled or even reparametrized in practice. Therefore, the protection devices are shutting off the PV plants for frequency drops below $47.5 \mathrm{~Hz}$ or gradually reducing the power for frequencies above $50.2 \mathrm{~Hz}$ before shutting off at $51.5 \mathrm{~Hz}$. This poses one of the greatest challenges for the grid restoration process of a distribution grid. Frequencies below $48 \mathrm{~Hz}$ are usual due to a low share of rotating masses. With the aforementioned protective shutdowns of PV plants the effective load step is even larger and can lead to instabilities or a new blackout. [9-11]

Moreover, most circuit breakers in distribution grids are not remotely controllable. In order to be able to switch the circuit breakers, personnel is required who have to drive long distances to reach the substations. Additionally, a stable and reliable communication needs to be assured between the control center and the personnel in the field.

In this case, voltage instability is of no concern during the grid restoration process of a distribution grid, frequency stability is the primary criterion [12]. Therefore, sophisticated control schemes are necessary to assure a participation of DERs in order to maintain the frequency and avoid critical drops. However, these should be designed without the need of communication between the DERs. [12]

Moreover, reliable load forecasts for switchable feeders are not possible due to several uncertainty factors. Therefore, worst-case scenarios with, among others, cold load pick-up (CLPU) and the magnetization currents of transformers need to be considered. However, it is also important to consider how even the load and RES are distributed in the feeders. [13]

While transmission system operator have a high observability of their grid and measurements of every line and substation, DSOs have not the necessary technology installed in their grid. Moreover, to assess the blackstart capability, indications are needed to be made based on data, which is easily accessible.

Moreover, in Europe a grid restoration process for distribution grids is mainly for rural distribution grids with high shares of RES. However, these grids are also having long feeders and often imbalanced load and PV power in these feeders. This can pose a significant challenge if e.g. feeders with high loads are reconnected in a later stage of the restoration process.

\section{Key indicators to classify distribution grids}

To address the aforementioned challenges in a distribution grid restoration, key indicators to classify a distribution grid regarding the capability of a restoration process are introduced in this chapter. An overview of the key indicators is given in table 1 .

First, the main question is if to build up the grid centralized from one blackstart unit or decentralized with more BSUs. Therefore, the commanding DER with $\mathrm{P}_{\mathrm{BSU}}$,high, which is often a CHP plant or a hydro power plant with the benefit of rotating masses and blackstart capability, is of great importance. This key indicator is telling two things regarding the grid restoration:

- Depending on the types of the Commanding DERs the maximal permissible load step and with this

- if the Commanding DER is capable to restart the grid from its connection point or if a decentralized grid restoration should be approached

$$
\text { ComDer }=\frac{P_{\text {BSU,high }}}{P_{\text {load,max }}} \text {. }
$$

Another important indication is the homogeneity of the distributed load and PV systems in the individual, with circuit breaker separated, feeders. To calculate the load balance

$$
\text { LoadBal }=1 / \frac{\text { Span }}{I Q} * g .
$$

a analysis of the connected loads in the grid areas is necessary. Therefore, the range of the load values is divided by the interquartile distance IQ. The reciprocal of this is then multiplied by the skewness $g$ of the load data set. This gives an indication regarding the expected homogeneity of the grid areas depending the load and is important to perform further investigations. These could identify critical grid areas where sophisticated strategies should be applied, or on the other hand, small grid areas which could be reconnected simultaneously.

As it is described in section 2, PV plants can become a challenge during a distribution grid restoration process. Therefore, the PV balance in the grid areas is another important factor in defining the best switching sequence. The PV balance is calculated analogous to the load balance with

$$
P v B a l=1 / \frac{S p a n}{I Q} * g .
$$

Another substantial factor in a grid restoration process is the speed of the grid restoration. Since distribution grids are not as remotely controllable as transmission systems, it is important to consider the remote controllable circuit breakers

$$
\mathrm{ReCBC}=\frac{\mathrm{n}_{\mathrm{cb}, \mathrm{ctrl}}}{\mathrm{n}_{\mathrm{cb}}} * \mathrm{n}_{\text {gridarea }}
$$


Table 1. Definition of the developed key indicators

\begin{tabular}{|c|c|}
\hline Commanding DER (ComDer) & Nominal power of the largest DER for an indication of permissible load steps \\
\hline Load Balance (LoadBal) & Homogeneity of the grid areas regarding the maximal active power of connected loads \\
\hline PV Balance (PvBal) & Homogeneity of the grid areas regarding the installed PV power \\
\hline Propotion of PV plants (ProPV) & The share of the installed PV power in relation to the total installed DER power \\
\hline $\begin{array}{l}\text { Remote circuit breaker controllability } \\
\text { (ReCBc) }\end{array}$ & The share of switchable grid areas via remotely controllable circuit breakers \\
\hline $\begin{array}{l}\text { Restoration process controlled } \\
\text { poweravailabillity (ResPow) }\end{array}$ & $\begin{array}{l}\text { Available controllable DER power for the grid restoration process (e.g. CHP plants, } \\
\text { hydro power plants or large wind turbines) }\end{array}$ \\
\hline Storage Ratio (StoRat) & The ratio of installed energy storage power with regard to the maximal load \\
\hline Non-fluctuating DER share (NonFlu) & The ratio of non-fluctuating installed power \\
\hline
\end{tabular}

Especially in distribution grids with large supply areas it is important to increase remote controlled circuit breakers since the alternative is that personnel needs to drive to the circuit breakers which can take up to 30 minutes for each switch.

As described, it is not practical to control every small DER. Hence, the ResPow

$$
\text { ResPow }=\frac{\mathrm{P}_{\mathrm{DER}, \text { ctrlable }}}{\mathrm{P}_{\text {load,max }}}
$$

indicates the controllable DER power. Depending on the grid, this can be extended to non-fluctuating controllable DER power.

It is not only important to be able to restore the grid but also to operate the grid in an island grid mode following a grid restoration. Therefore, it is evenly important to have some form of energy storage. This could be a BESS, which is able to help during the restoration process with providing power faster than e.g. CHP plants. Moreover, BESS can provide important balance power for the volatility of PV plants or wind turbines. This is necessary during the blackstart as well as the island grid operation. Therefore, the ratio of the available storage power is defined by

$$
\text { StoRat }=\frac{\mathrm{P}_{\text {stor }}}{\mathrm{P}_{\text {load,max }}} .
$$

Lastly, it is important to assess the share of nonfluctuating DERs like CHP plants, hydro power plants or BESS with

$$
\text { NonFlu }=\frac{\mathrm{P}_{\text {stor }} \mathrm{P}_{\mathrm{CHP}+} \mathrm{P}_{\text {hydro }}}{\mathrm{P}_{\text {load, } \max }} .
$$

Obviously, a distribution grid restoration is highly dependent on fluctuating energy resources. Therefore, with this indicator the possibility to restore a grid with changing weather conditions is given. However, further considerations and distinctions between solar and wind power should be made.

\section{Study case of a German distribution grid}

In this chapter, an approach for a restoration process in a distribution grid is evaluated. First, the main challenges of a distribution grid restoration process are explained. Next, the methodology for the proposed restoration process will be introduced.

\subsection{Specifics of the present distribution grid}

In this case study, a German distribution grid has been investigated. It supplies 18,000 customers with a cable length of $170 \mathrm{~km}$ in the medium voltage and $260 \mathrm{~km}$ in the low voltage grid. Moreover, it is divided in eleven feeders. In 2018, the annual peak load was $13.34 \mathrm{MW}$.

In general, the grid is meshed. However, due to the high protection requirements of a meshed network, it is not operated as such, but rather as an open ring system. It is possible to control larger generation plants remotely. This allows the system operator to control the active power of larger DERs to assure a reliable and stable island grid operation and restoration.

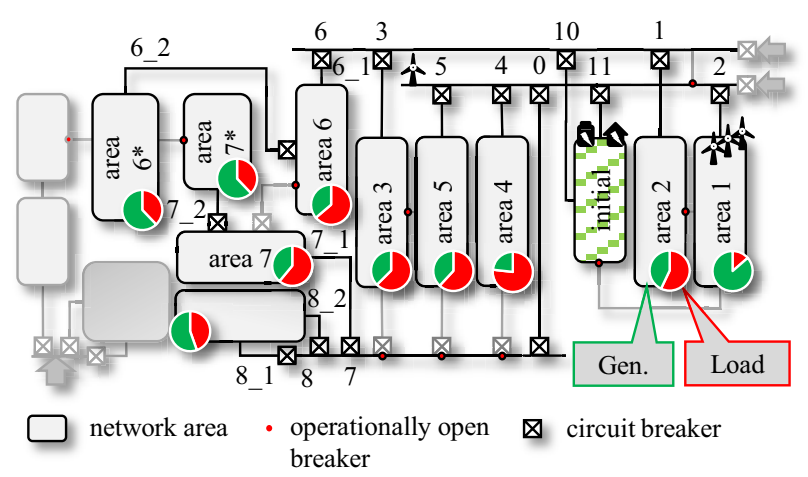

Fig. 1. Schematic plan of the considered medium voltage grid

The distribution grid in this case study has a high penetration of RES. A significant part of the installed generation capacity is located in the city centre (area 3-5) and the urban areas of 1-2 and 7 (cf. Fig. 1). Notably, this 
includes a 13.5 MW CHP plant and an 8 MVA BESS displayed with initial. Additionally, a 1.2 MW black start capable CHP plant is connected to the same common point of coupling.

Moreover, four wind power plants with an installed capacity of approximately $10 \mathrm{MWp}$ are located at different connection points in feeder two. Throughout the grid, about $700 \mathrm{PV}$ systems with a maximum peak power of $11 \mathrm{MWp}$ are connected to the low voltage $(\sim 95 \%)$ and the medium voltage level ( $\sim 5 \%)$. Moreover, almost $93 \%$ of the installed PV systems are household systems with less than $50 \mathrm{kWp}$. Especially these PV plants are not evenly distributed in the grid and are not controllable from an economic point of view. Therefore, these PV plants are primarily responsible for the challenges that are described in section 2.

\subsection{Key indicators of the case study}

In the present case study, the developed indicator system is shown in figure 2. The corresponding input data and values of the key indicators are shown in table 2.

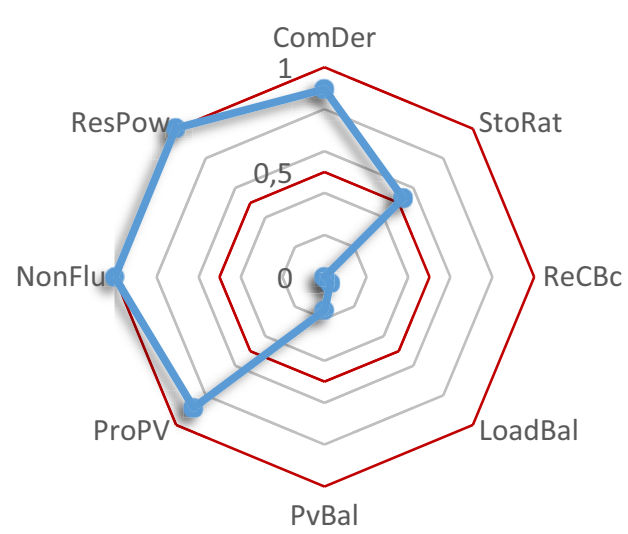

Fig. 2. Key indicators systems for the considered use case

As it can be seen, the chosen example grid has a strong characteristic towards the left part of the radar chart. Hence, the value for ComDer is with 0.9 exceptional high. This shows, that the commanding DER can supply almost $90 \%$ of the maximal load. Obviously, the large CHP plant for an industrial application is of great use for restoring this grid. Not only that, but also a StoRat of 0.53 is an indication for the capability to be able to restore the grid. Another positive indicator is the ResPow which shows that the power of controllable power plants is above the maximal load $\mathrm{P}_{\max }$.

However, the weak manifestation of the lower left part of the spider chart shows on the one hand highly imbalanced feeders regarding both, the installed PV power and the load. This shows that the installed PV power is not evenly distributed through the feeder. The same applies to the distribution of the loads. Hence, a stable grid restoration even with the large commanding DER cannot be assured.
What's more, the ReCBc shows with 0 that the circuit breakers are not remotely controllable. This results in potentially long waiting periods between the switching actions. However, the distance between the switches should be taken into account here.

The ratio of PV plants (ProPV) together with the imbalanced distribution in the feeders show a significant influence of the PV plants. Hence, a grid restoration by night could be difficult while a strategy for minimizing their negative impact on the grid during a blackstart needs to be considered.

Concluding, the non-fluctuating DERs are with a share of over $100 \%$, especially due to the non-fluctuating commanding DER, well equipped to restore the grid independently of the weather situation.

Table 2. Key indicators for the considered case study

\begin{tabular}{c|c} 
Commanding DER ( ComDer) & $\mathbf{0 . 9 0}$ \\
\hline Load Balance (LoadBal) & $\mathbf{0 . 0 3}$ \\
\hline PV Balance (PvBal) & $\mathbf{0 . 1 6}$ \\
\hline Proportion of PV plants (ProPV) & $\mathbf{0 . 8 8}$ \\
\hline $\begin{array}{c}\text { Remote circuit breaker controllability } \\
\text { (ReCBc) }\end{array}$ & $\mathbf{0 . 0}$ \\
\hline $\begin{array}{c}\text { Restoration process controlled } \\
\text { poweravailabillity (ResPow) }\end{array}$ & $\mathbf{1 . 0 1}$ \\
\hline Storage Ratio (StoRat) & $\mathbf{0 . 5 3}$ \\
\hline $\begin{array}{c}\text { Non-fluctuating DER share (NonFlu) } \\
\text { Pow }\end{array}$
\end{tabular}

\subsection{Concluding grid restoration strategies}

With the key indicators of the use case from section 4.2, various restoration strategies for the considered grid can be developed and derived.

Here, four conclusions can be made straight away. First, the remote controllability of the circuit breaker is a significant feature of a stable and reliable grid restoration. Therefore, a meaningful investment for the distribution system operator for a stable and reliable grid restoration is exposed.

Moreover, the ComDer and StoRat values are showing that coordinated control schemes between the commanding DER and the storage systems could be beneficial. Hence, innovative control strategies for grid restoration processes with CHP plants and BESS should be applied here. Moreover, a centralized grid restoration process should be considered in this case.

The most critical feature of this grid is the imbalance in installed PV power and the load distribution. Therefore, even with the large commanding DER, critical load steps are possible. However, feeders with low load share can be connected faster or simultaneously. To minimize the load step of the critical feeders, demand side management or 
the coordination with commercial/industrial customers in the corresponding feeder are necessary.

As explained in section 2, PV plants can complicate the grid restoration during the application of the load. Here, the share of PV plants is considerable high. Since the change of the control scheme of every small PV plant is not practical, strategies like a $52 \mathrm{~Hz}$ Strategy should be taken into account. Especially in the considered use case, the non-fluctuating installed power is sufficient to provide the necessary power during a grid restoration. Therefore, the commanding DER could operate the grid at a frequency of $52 \mathrm{~Hz}$ where the PV plants would not be reconnecting to the grid. This would also lead to higher frequency NADIRs. After successful grid restoration, the frequency could be decreased to the nominal value $f_{\text {nom }}$.

\section{Conclusion and outlook}

In this paper, a key indicator system, especially focusing on the grid restoration of distribution grids, has been developed. These indicators, like the ratio of the commanding DER in regards to the maximal annual load (ComDer) or the homogeneity of loads and installed PV power in the feeder of a distribution grid (PvBal, LoadBal), are aimed to lucidly displaying the key information to evaluate a distribution grid regarding a grid restoration and narrow down possible restoration strategies. Therefore, the main challenges of a distribution gird restoration after a large scale blackout are defined and analyzed. The developed key indicators are addressing these challenges with the use of accessible data from distribution grid operators. However, not all data are publicly available. Especially the number of grid areas and the load and PV distribution in the feeders.

The main focuses in this work are the significant impact of PV plants, investment recommendations for DSOs and the applicability into the practice. Investment recommendations are given in particular for the digitalization of the distribution grid and the investment in BESS. The applicability into the practice aims primarily at the feasibility of the control schemes and the suggested restoration strategies. However, the indicator system just gives a broad overview of the capability to blackstart the grid.

In future works, a pre-analysis based on a grid model in a power system analyzation software should be developed to specify the restoration strategy in more detail. Therefore, load and RES balances in the feeder can be monitored and valued. However, while a pre-analysis can be load flow based, dynamic investigations need to be performed to assess the real behavior during a distribution grid blackstart.

\section{References}

1. L. Seca, H. Costa, C. L. Moreira, J. A. P. Lopes, An innovative strategy for power system restoration using utility scale wind parks, IREP Symposium Bulk Power System Dynamics and Control, (2013)

2. H. Becker, T. Henning, A. Akbulut, D. Mende, L. Hofmann, Netzwiederaufbaukonzepte: Moegliches Zusammenspiel zwischen Windenergieanlagen und thermischen Kraftwerken, VGB PowerTech, 10, pp. 57-62 (2016)

3. Community Research and Development Information Service (CORDIS): The Cost of Blackouts in Europe, Online, cordis.europa.eu/news/, (2019)

4. C. Strunck, M. Albrecht, G. Meindl, C. Rehtanz: A Study on the Black Start Process of a real Distribution Network with CHP plants and BESS, doi: 10.1051/epjconf/201921701015, (2019)

5. W. Sun, C. Liu and S. Liu, Black start capability assessment in power system restoration, 2011 IEEE Power and Energy Society General Meeting, Detroit, MI, USA, (2011), pp. 1-7, doi: 10.1109/PES.2011.6039752.

6. Kai Sun; Yunhe Hou; Wei Sun; Junjian Qi, BlackStart Capability Assessment and Optimization, in Power System Control Under Cascading Failures: Understanding, Mitigation, and System Restoration, IEEE, (2019), pp.399-432, doi: 10.1002/9781119282075.ch10.

7. W. Heckmann, et. al., NETZKRAFT Netzwiederaufbau unter Berücksichtigung zukünftiger Kraftwerkstrukturen, (2019)

8. European Comission, Distribution System Operators Observatory, (2016)

9. ENTSO-E, Continental Europe Operation Handbook - Policy 5: Emergency Options, (2017)

10. VDE Verlag GmbH, Connection and Operation of Power Generating Plants to Low-Voltage Grid (VDE-AR-N 4105), (2018)

11. VDE Verlag GmbH, Technical requirements for the connection and operation of customer installations to the medium voltage network (TAR medium voltage), (2018)

12. M. Farrokhabadi et al., Microgrid Stability Definitions, Analysis, and Examples, in IEEE Transactions on Power Systems, vol. 35, no. 1, pp. 13-29, (2020), doi: 10.1109/TPWRS.2019.2925703.

13. A. M. El-Zonkoly, Power system single step restoration incorporating cold load pickup aided by distributed generation, (2012) 